\title{
Low co-existence rates of Lactobacillus spp. and Helicobacter pylori detected in gastric biopsies from patients with gastrointestinal symptoms
}

\author{
Apolinaria García ${ }^{1}$, Katia Sáez ${ }^{2}$, Carolina Delgado ${ }^{3}$ and Carlos L. González ${ }^{1}$ \\ ${ }^{\prime}$ Bacterial Pathogenicity Laboratory. Department of Microbiology. School of Biogical Sciences. ${ }^{2}$ Department of Statistic. \\ School of Physical and Mathematics Sciences. ${ }^{3}$ Molecular Pathology Laboratory. Department of Medical Specialties. \\ School of Medicine. University of Concepcion. Concepcion, Chile
}

\begin{abstract}
Background: bacterial diversity of the stomach includes various species. Among them, Helicobacter pylori, a microorganism which has been associated to gastric diseases, is frequently isolated in this habitat. In addition, Lactobacillus spp., a genus including probiotic strains, has also been documented in this habitat. The co-existence of these two species in the stomach of symptomatic patient needs to be elucidated.

Aims: our goal was to establish if Lactobacillus spp. and $H$. pylori co-exist in the stomach mucosa of symptomatic patients.

Methods: gastric biopsies (antrum and/or the body) from 427 Chilean patients with gastrointestinal discomfort were analyzed. The $H$. pylori infection and/or Lactobacillus spp. colonization status was determined for each patient by standard culture techniques, and statistical correlations between the presence of those species and the age, gender, or the severity of the gastric disease were also established.

Results: only $6.1 \%$ of the samples presented co-existence of Lactobacillus spp. and $H$. pylori. This former species was isolated in $42.6 \%$ of the patients as unique species, while Lactobacillus spp. was isolated as single species in $19.4 \%$ of the individuals. Chronic non-atrophic gastritis was prevalent in Lactobacillus spp. non colonized individuals, while chronic non-atrophic and chronic atrophic gastritis diagnosis was similar in Lactobacillus spp. harbouring individuals $(p<0.001)$. The presence of Lactobacillus spp. significantly increased with age $(p=0.005)$, independently of gender.

Conclusion: the negative Pearson correlation between Lactobacillus spp. and $H$. pylori $(r=-0.112, p=0.020)$ indicates that the co-existence of both species is low in human gastric mucosa of symptomatic patients.
\end{abstract}

Key words: Lactobacillus. Helicobacter. Co-existence. Gastric mucosa.

Received: 12-06-2012

Accepted: 11-09-2012

Correspondence: Apolinaria García. Departamento de Microbiología. Facultad de Ciencias Biológicas. Universidad de Concepción. Casilla 160-C. Concepción-Chile

e-mail: apgarcia@udec.cl
García A, Sáez K, Delgado C, Carlos L. González. Low co-existence rates of Lactobacillus spp. and Helicobacter pylori detected in gastric biopsies from patients with gastrointestinal symptoms. Rev Esp Enferm Dig 2012;104:473-478.

\section{INTRODUCTION}

Human microbiota in both healthy and non-healthy individuals has greatly incited the interest of the medical science research community. Molecular techniques have demonstrated that the bacterial biodiversity of various sites, including stomach, were underestimated by classic microbiological techniques $(1,2)$. Gastric microbiota has been primarily characterized by the culture of gastric biopsies and/or gastric secretion $(3,4)$. Molecular techniques have enabled a more detailed study of this ecosystem, resulting in the detection of 128 different phylotypes, and the identification of species belonging to the Firmicutes, Proteobacteria, Actynobacteria and Fusobacteria phyla and yeast. Among these, approximately $10 \%$ were newly described bacterial species in the human stomach $(1,5,6)$. One member of this microbiota is $H$. pylori, a species considered to be a promoting agent of severe gastric diseases, such as peptic ulcer, mucosa associated lymph tissue (MALT) lymphoma and gastric cancer $(7,8)$.

The genus Lactobacillus is one of the main genus containing probiotic strains (9-11) which are acid-resistant (12). Probiotics are live organisms that are orally administrated, usually in addition to conventional antibiotic therapy. They may modulate the human microbiota and promote health, prevent antibiotic side effects, stimulate the immune response and directly compete with pathogenic bacteria (13). They could be present in this acidic gastric environment (14). However, the ability of these species to either 
co-exist or to compete in the gastric habitat remains to be established. Possible mechanisms include inhibition of $H$. pylori urease enzyme, disruption of bacterial cell membrane, and modulation of the host immune system (13).

We hypothesize that these two species are, in general, mutually exclusive in the human gastric stomach mucosa based on the antagonism showed in vitro by several strains of Lactobacillus spp. against $H$. pylori.

Our goal was to determine the presence of both Lactobacillus spp. and $H$. pylori in the stomach of Chilean patients that exhibited gastrointestinal symptoms, in order to establish the ability of these two species to co-exist or compete. The correlation between gastric damage, age, gender and the prevalence of these two species in the stomach were also investigated.

\section{METHODS}

\section{Samples}

This study is part of a larger analysis designed to determine the prevalence of $H$. pylori infection in Chile, the resistance phenotype of the clinical isolates toward first line and second line antibiotics used in eradication therapy, and the search for new anti $H$. pylori active compounds (probiotics and phytopharms).

\section{Patients}

Four hundred and twenty-seven patients, children and adults with gastric disorders, recruited between years 20052007 were included. Patients were $60 \%$ women (256 individuals) and $40 \%$ men (171 individuals), and were distributed exclusively according to age in four groups, as follows: group A, patients under age 10 (11 individuals); group B, patients between ages 10-19 (42 individuals); group C, patients between ages 20-64 (237 individuals); and group D: over age 64 (54 individuals). Eighty three patients were not considered in any group because none age data was available. All patients included in this study signed a consent form for tissue organ donor, previously approved by the Ethics Committee of the School of Medicine at the University of Concepción, Chile. Individuals were fasted for at least 12 hours prior to the gastric endoscopic procedure. Vaira et al. (15) criteria for patients exclusion was used which considers at least four months without $\mathrm{H} 2$ antagonists or proton pump inhibitors intake to be eligible when serological analysis are included.

\section{Isolation and identification of the species}

Two gastric biopsies per patient that were obtained from the antrum or the body of the stomach were homogenized in a mortar and inoculated in Columbia agar supplemented with $5 \%$ of horse blood and DENT (Oxoid), at $37{ }^{\circ} \mathrm{C}$ under microaerobic atmosphere for 5 days to isolate $H$. pylori. Identification was made using Gram stain, urease and catalase tests. Lactobacillus spp. was isolated by pre-incubating $0.1 \mathrm{ml}$ of the above homogenate in $1 \mathrm{ml}$ of MRS broth at $37{ }^{\circ} \mathrm{C}$ under $10 \% \mathrm{CO}_{2}$ atmosphere for 2 days, followed by subculture in MRS agar. The isolates were further identified by using Gram stain, catalase and oxidase tests.

Molecular identification of $H$. pylori was carried out using conventional PCR with primer-specific for Hpy-F (5' -TGCGAAGTGGAGCCAATCTT-3') and Hpy-R (5'-GGCCCGTATTCACCGCAACA-3'), that renders a 199 bp PCR product from the $16 \mathrm{~S}$ rRNA gene (16). Lactobacillus spp. was identified using conventional PCR and API $50 \mathrm{CH}$ kit (Biomerieux) with specific primers for Lac-F (5'-GAATCGCTAGTAATCG-3') and Lac-R (5'-GGGTTCCCCCATTCGGA-3'), that renders a 186 bp PCR product from the hypervariable $16 \mathrm{~S}$ - 23S rRNA region. BLAST sequencing analysis was used to identify the group of $L$. rhamnosus, $L$. casei, L paracasei, L. zeae and L. curvatuns (17). Primers LbG-F (5'-AGAAGAGGACAGTGGAAC-3') and LbG-R (5'-TTACAAACTCTCATGGTGTG-3') that generate a 750 bp PCR product were used to identify L. brevis, $L$. casei, $L$. curvatus, L. fermentum, L. harbinensis, L. hilgardii, L. kefiri, L. kunkeei, L. parabuchneri, L. paracasei, L. plantarum, L. pentosus, L. sakei, L. salivarius, L. sanfranciscensis as another bacterial group (10).

\section{Histopathological analysis}

Samples for histopathology were processed, as described elsewhere, using the Sydney classification (18). Only those samples with consensus for the histopathological diagnosis were considered in this study.

\section{Statistical analysis}

The results were analyzed by Chi-square test, with confidence intervals of $95 \%$, using the Statistical Package for Social Science software (SPSS), version 10.0.

\section{RESULTS}

The lactobacilli strains isolated from the gastric biopsies were presumptively identified as belonging to the genus Lactobacillus by classical microbiological methods, and were further confirmed as belonging to one of the two lactobacilli groups described in Material and methods, by using conventional PCR. In addition, the first 21 isolated lactobacilli strains were also identified at the species level by using the API 50 CHL kit. Ten strains were identified as L. fermentum, three strains were identified as L. rhamnosus, three strains were identified as L. salivarius, one strain was identified as $L$. paracase $i$, and one strain was 
identified as L. plantarum. On the other hand, three strains were not possible to be typified with this kit.

Among the 427 patients analysed, $H$. pylori was isolated from $42.6 \%$ while Lactobacillus spp. was isolated in $19.4 \%$ of individuals. Thirty six and a half percent of the total individuals presented only $H$. pylori in their gastric mucosa meanwhile $13.4 \%$ showed Lactobacillus spp. as single species. Interestingly, both species were found simultaneously in $6.1 \%$ of the patients (26 individuals), and 188 individuals were free of both $\mathrm{H}$. pylori and Lactobacillus spp. (44\% of patients). A negative correlation $(\mathrm{r}=-0.112)$ between these two microorganisms was detected, because 156 patients out of 182 were infected with $H$. pylori but were free of Lactobacillus spp. (85.7\% of individuals). On the other hand, 57 of 83 patients colonized by Lactobacillus spp. were free of $H$. pylori ( $68.7 \%$ of individuals).

Histological diagnosis showed that $2.8 \%$ patients (12/427 individuals) had a normal gastric mucosa, $72.8 \%$ of patients presented chronic non-atrophic gastritis (Cnon-AG) (311/427 individuals), 23.7\% had chronic atrophic gastritis (CAG) (101/427 individuals) and $0.7 \%$ had gastric carcinoma (3/427 individuals). H pylori infected and non infected individuals ratio was similar in both Cnon-AG patients -ratio 0.77 (135/176 individuals)- and CAG -ratio 0.8 (45/56 individuals)-(Fig. 1A). None of the individuals with normal mucosa were infected by $H$. pylori while this species was isolated from the gastric mucosa of two cancer patients (2/3 individuals). Similar analysis considering Lactobacillus spp. colonized patients as reference, showed that the colonized and non-colonized patients ratio was 0.17 (46/265 individuals) in Cnon-AG patients and 0.51 (34/67 individuals) in CAG patients (Fig. 1B). On the other hand, two of 12 patients with normal mucosa were colonized by Lactobacillus spp. and this species was also isolated from the mucosa of one of the tree cancer patients.

A statistically significant correlation was observed between Cnon-AG or CAG patients and the presence of $H$. pylori $(\mathrm{p}=0.02)$ or Lactobacillus spp. $(\mathrm{p}<0.001)$ in their gastric mucosa. Thus, H. pylori was isolated in similar proportion from the gastric mucosa of individuals with diagnosis of both Cnon-AG and CAG, but Lactobacillus spp. was isolated mainly from CAG patients $(\mathrm{p}<0.001)$ (Fig. 1C).

Age of individuals also showed a correlation with the severity of gastric lesion and the status of Lactobacillus spp. colonization (Fig. 2). None of the individuals under age 10 presented CAG or gastric cancer, but an increasing number of CAG patients was observed in group B $(2.4 \%$ of individuals), group C (26.2\% of individuals), and group $\mathrm{D}$ (38.9\% of individuals). On the other hand, gastric cancer patients were only detected in $2 / 237$ patients of group C ( $0.84 \%$ of individuals) and $1 / 54$ patients of group D ( $1.9 \%$ of individuals). Patients under 10 years old were not colonized by Lactobacillus spp. (Fig. 2A), but a statistically significant increase in Lactobacillus spp. colonization among individuals included in all the other groups was observed $(\mathrm{p}=0.005)$, with prevalence of $4.8 \%$ in group $\mathrm{B}$ (Fig. 2B), $19.4 \%$ in group C (Fig. 2C), and $31.5 \%$ in group

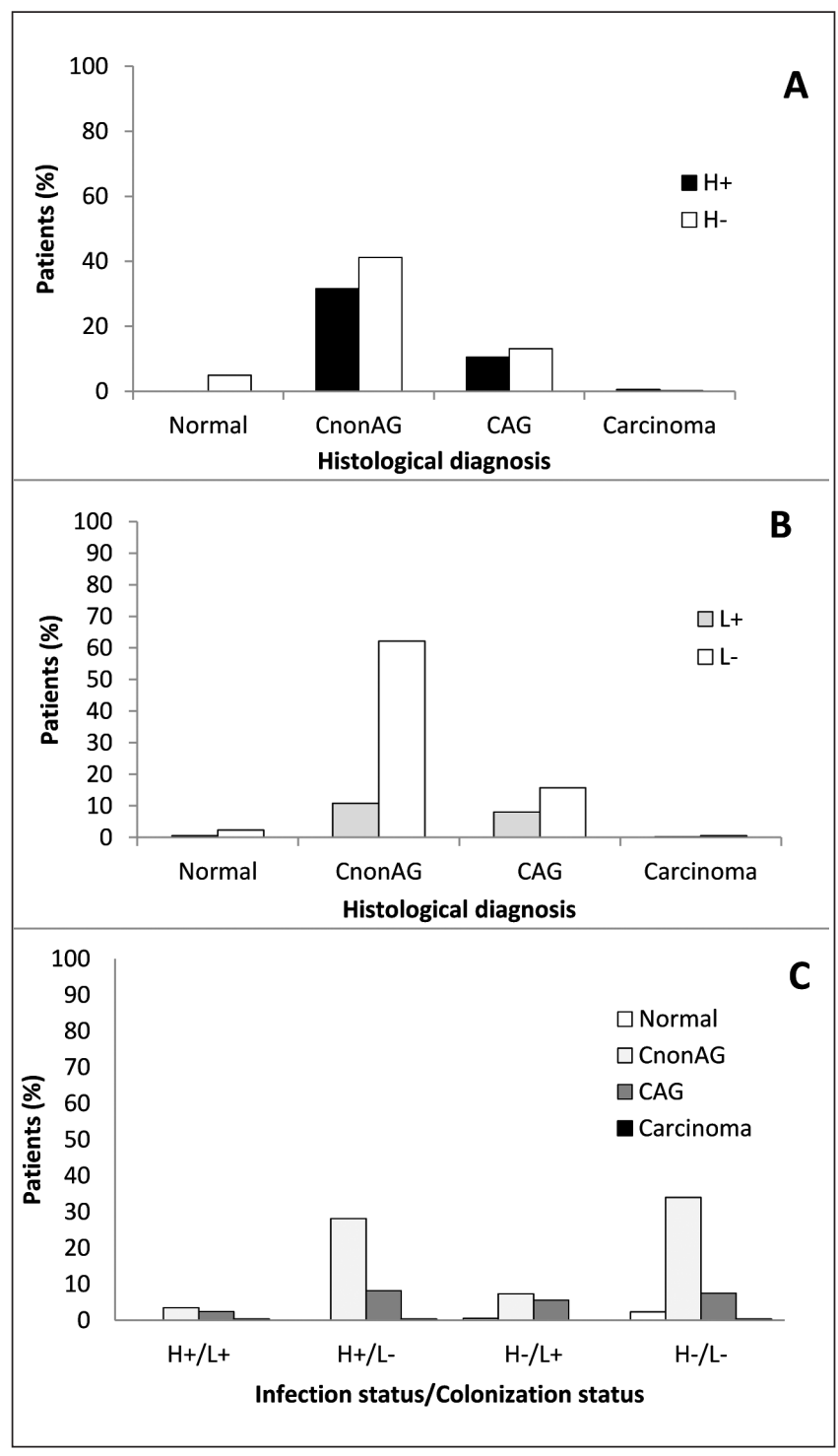

Fig. 1. Relationship between Helicobacter pylori or Lactobacillus spp. status and histological diagnosis. A. Helicobacter pylori infection status and histological diagnosis. B. Lactobacillus spp. colonization status and histological diagnosis. C. Presence of both Helicobacter pylori and/or Lactobacillus spp. and histological diagnosis $(\mathrm{H}$ : H. pylori, L: Lactobacillus spp.; CAG: chronic atrophic gastritis; and Cnon-AG: chronic non-atrophic gastritis).

D (Fig. 2D) was observed. Prevalence of infection with $H$. pylori also showed positive correlation with age, from $18.2 \%$ in patients under 10 years old to $48.5 \%$ in patients belonging to group $\mathrm{C}(\mathrm{p}=0.017)$, but this correlation was lost in elderly patients (over 64 years old) were a prevalence of $29.6 \%$ was detected.

Finally, H. pylori infection or Lactobacillus spp. colonization status showed no significant differences with gender because $H$. pylori was isolated from $41 \%$ of women (105/256 individuals) and $45 \%$ of men (77/171 individuals), meanwhile Lactobacillus spp. was isolated from $20.3 \%$ of women (52/256 individuals) and $18.1 \%$ of men (31/171 individuals). 


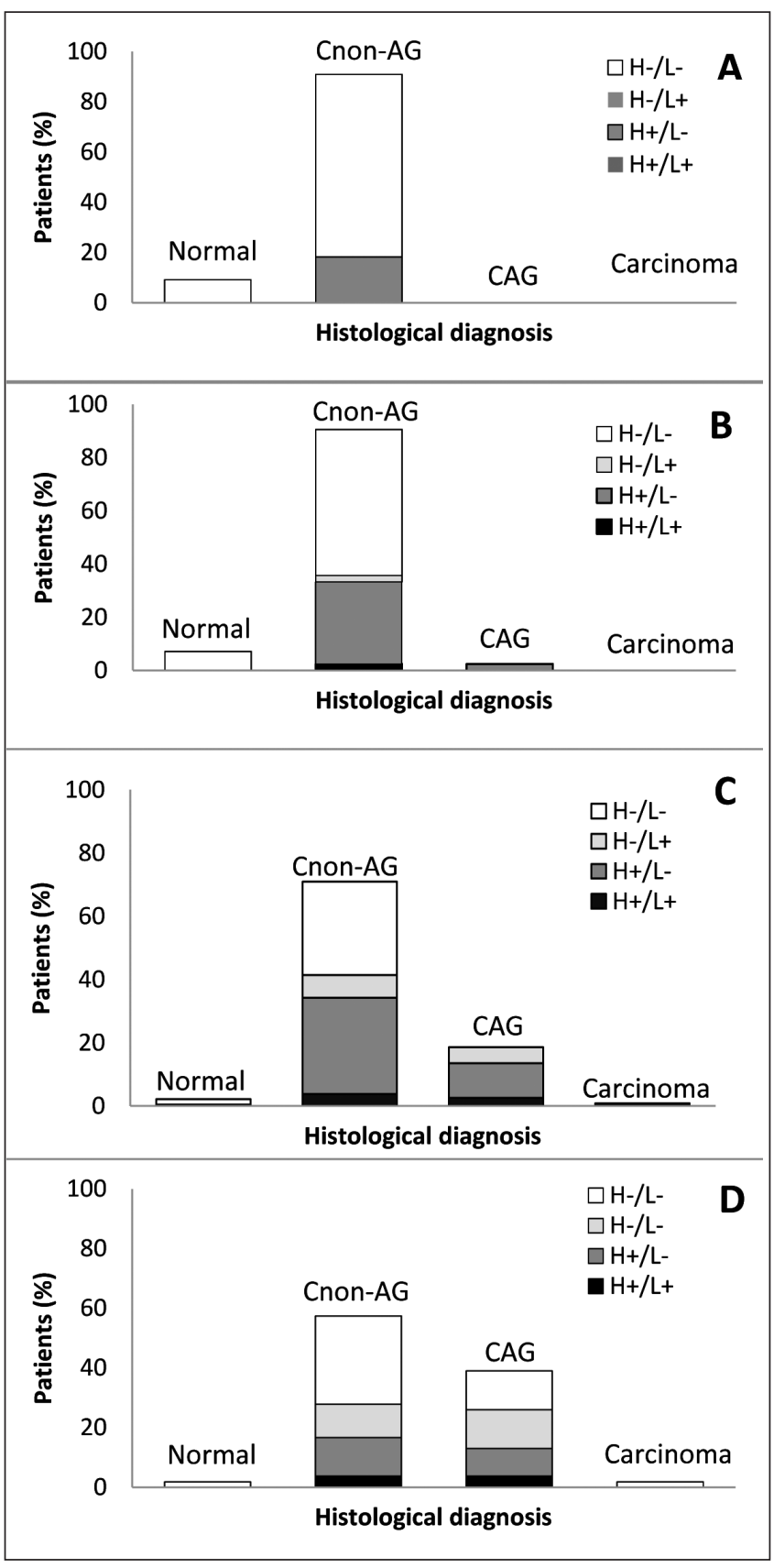

Fig. 2. Relationship between Helicobacter pylori or Lactobacillus spp. status and age. Patients were distributed in four age groups (A-D), and investigated for the presence of both bacterial species: A. Patients under age 10. B. Patients between ages 10-19; C. Patients between ages 2064; D. Patients over age 64 (H: H pylori; L: Lactobacillus spp.; CAG: chronic atrophic gastritis; CnonAG: chronic non-atrophic gastritis).

\section{DISCUSSION}

Risk factors allowing the infection of humans by $H$. pylori and persistence are still under investigation, because new evidence is needed to better understand the pathogenesis of disease's development due to this microorganism.
One of the factors that have attracted the attention to date in this theme is the role of co-colonization of the stomach by other bacterial species in gastric mucosa damage progress or in injury avoidance. Specifically, the role of Lactobacillus in the stomach of symptomatic individuals was addressed in this work to understand if this bacterial genus affects the evolution of $H$. pylori associated diseases.

Ten strains out of the first 21 strains analyzed by API50 CHL kit were identified as L. fermentum, and this species has been isolated with increased frequency from adenocarcinoma (5/9 patients) and benign gastric ulcer ( $5 / 8$ patients) with an overall frequency of $44.8 \%$ (13/29 patients) (19). Our results are consistent with this finding because $47.6 \%$ (10/21 individuals) of patients were colonized by L. fermentum as the main species.

Lactobacillus spp. was isolated as single bacterial species in $10 \%$ of the patients with Cnon-AG (31/311 patients), and in $23.8 \%$ of CAG patients (24/101 patients). These results suggest that Lactobacillus spp. colonise the gastric mucosa of individuals previously injured by various conditions, including gastric cancer (2). The alkaline $\mathrm{pH}$ of the stomach in these patients or an increased exposure of the epithelial cells to the microorganisms due to mucosa damage could favour the growth of Lactobacillus spp. Although several studies indicate that Lactobacillus spp. colonisation diminish gastric inflammation, promote mucin regeneration and down-regulate several genes of the cag pathogenicity island (20,21), one study (19) speculates that Lactobacillus spp. might play a role in malignancy as they can be isolated from the gastric mucosa of patients with adenocarcinoma.

Medical records detailing the gastric history of patients participating in our study were not available. However, participants were considered to be long term infected by $H$. pylori because Chilean population acquire bacterial infections during early childhood (22), and over $70 \%$ of the adult population with gastric disorders harbors $H$. pylori as detected by serological analysis in a large population study $(2,615$ individuals) (23). Our results are based on culturing recoverable bacteria, which is the recommended test when antibiotic resistant pattern of clinical isolates should be determined, even though this method is less sensitive for detecting infected individuals than serology (24). The $42.6 \%$ of prevalence informed by us is similar with the results published by Otth et al. (25) for a Chilean cohort $(41.3 \%)$. Thus, short-term infection among Chilean patients is rather scarce. Furthermore, H. pylori infection may persist for many decades prior to the outbreak of gastric disturbance (26), and subjects with decreased acid output usually show also atrophy in their gastric epithelium, with progression to multifocal metaplasia (27). Whilst these patients fail to present specific symptoms, their risk for gastric cancer development is increased 5- to 90-fold, depending upon the extension and severity of atrophy (27).

Moderate to severe inflammation found by histopathological studies of gastric biopsies suggests infection by type I strains of H. pylori (28). Nonetheless, previous results published by our research group (29) indicate that 
some strains of type II H. pylori (genotype $s 2 m 2$ ) may harbour specific type of LPS that induce pro-inflammatory cytokines in mice model, suggesting also a potential role of these strains in human gastric inflammation. Chilean clinical isolates of $H$. pylori are usually cagA positive, vacAsl and vacAml (30), therefore, type I strains of $H$ pylori may be partially responsible for the histopathological finding among Chilean patients. However, we cannot rule out the possibility that simultaneous infection with more than one genotype of $H$. pylori could play a role in damage severity, because it has been seen that a fraction of Chilean population is infected by two or more $H$. pylori genotype (31).

Interestingly, our results indicate that $H$. pylori and Lactobacillus spp. do not co-exist in the gastric mucosa $(\mathrm{p}=$ 0.02 ), suggesting a mutually exclusive behaviour of these two bacterial species for colonizing the stomach mucosa. Moreover, colonization may be attributed to chronic inflammation of the stomach due to $H$. pylori infection because a more suitable environment for Lactobacillus spp. development became available after long term infection as proposed by others $(2,32)$. However, Ryan et al. (33), searching for the probiotic activity of Lactobacillus spp., did not find correlation between Lactobacillus spp. colonization and gender, age, $H$. pylori infection, or pathology. However, their result can be attributed to the reduced study group which was analysed (12 patients). Our results suggest that age is important for Lactobacillus spp. colonization of the stomach, with a concomitant decrease of $H$. pylori infection prevalence in those individuals over age 65 . The results presented are based in larger group of individuals (427 patients), thus, the $19.4 \%$ of prevalence detected should be used as the reference value for Lactobacillus colonization of Chilean symptomatic individuals.

The newly described species of Lactobacillus, L. gastricus and $L$. antri in the subgroup $L$. reuteri, defined as members of the stomach resident microbiota, and which do not grow in MRS agar (14), increase the uncertainty of culture methods as searching tool for this gender in clinical samples. Thus, combined methods like PCR and conventional microbiological techniques should be used in future analysis to establish with more certainty an exclusive model of stomach colonisation for these two species. Nonetheless, the negative correlation observed should be maintained because cultures usually resemble the relative abundance of viable cells for Lactobacillus spp. and H. pylori in biological samples, especially if more than one biopsy samples per individual are used for searching of $H$. pylori and Lactobacillus spp. (34). More data are needed to confirm that long-term $H$. pylori infection is one of the main factors allowing Lactobacillus spp. colonization.

\section{ACKNOWLEDGEMENT}

This work was supported by Grant DIUC 208.036.0331.0, University of Concepcion, and Grant D03I 1105,
FONDEF - Chile. We thank Mrs. Maria E. Larenas for language review of the manuscript.

\section{REFERENCES}

1. Bik EM, Eckburg PB, Gill SR, Nelson KE, Purdom EA, Francois F. Molecular analysis of the bacterial microbiota in the human stomach. Proc Natl Acad Sci USA 2006;103:732-7

2. Dicksved J, Lindberg M, Rosenquist M, Enroth H, Jansson JK, Engstrand L. Molecular characterization of the stomach microbiota in patients with gastric cancer and in controls. J Med Microbiol 2009; 58:509-16.

3. Savage DC. Microbial ecology of the gastrointestinal tract. Annu Rev Microbiol 1977;31:107-33.

4. Adamsson I, Nord CE, Lundquist P, Sjöstedt S, Edlund C. Comparative effects of omeprazole, amoxicillin plus metronidazole versus omeprazole, clarithromycin plus metronidazole on the oral, gastric and intestinal microflora in Helicobacter pylori-infected patients. J Antimicrob Chemother 1999;44:629-40.

5. Hugenholz P, Goebel BM, Pace NR. Impact of culture-independent studies on the emerging phylogenetic view of bacterial diversity. J Bacteriol 1998; 180:4765-74.

6. Zoetendal EG, Collier CT, Koike S, Mackie RI, Gaskins HR. Molecular Ecological Analysis of the Gastrointestinal Microbiota: A Review. J Nutr 2004; 134:465-72.

7. Blaser MJ, Atherton J. Helicobacter pylori persistence: Biology and disease. J Clin Invest 2004;113:321-3.

8. Sachs G, Scott DR. Helicobacter pylori: Eradication or Preservation. F1000 Medicine Reports 2012;4:7.

9. Sullivan A, Nord C. Probiotics and gastrointestinal diseases. J Inter Med 2005;257: 78-92.

10. Kolida S, Saulnier DM, Gibson GR. Gastrointestinal microflora: probiotics. Adv Appl Microbiol 2006;59:187-219.

11. Saad N, Delattre C, Urdaci M, Schmitter JM, Bressollier P. An overview of the last advances in probiotic and prebiotic field. LWT - Food Science and Technology 2012; Available at: http://dx.doi.org/10.1016/ j.lwt. 2012.05.014.

12. Marteau P, Minekus M, Havenaar R, Huis In't Veld JH. Survival of lactic acid bacteria in a dynamic model of the stomach and small intestine: validation and the effects of bile. J Dairy Sci 1997;80:1031-37.

13. Vítor JM, Vale FF. Alternative therapies for Helicobacter pylori: probiotics and phytomedicine. FEMS Immunol Med Microbiol 2011; 63:153-64.

14. Roos S, Engstrand L, Jonson H. Lactobacillus gastricus sp. nov., Lactobacillus antri sp. nov., Lactobacillus kalixensis sp. nov. and Lactobacillus ultunensis sp. nov., isolated from human stomach mucosa. Int J Syst Evol Microbiol 2005;55:77-82.

15. Vaira D, Malfertheiner P, Mégraud F, Axon ATR, Deltenre M, Hirschl AM, et al. Diagnosis of Helicobacter pylori infection with a new noninvasive antigen-based assay. Lancet 1999;354:30-3.

16. Yamazaki S, Kato S, Matsukura N, Ohtani M, Ito Y, Suto H, et al. Identification of Helicobacter pylori and cagA genotype in gastric biopsies using highly sensitive real-time PCR as a new diagnostic tool. Fed Eur Microbiol Soc Immunol Med Microbiol 2005;44:261-8.

17. Tilsala-Timisjärvi A, Alatossava T. Development of oligonucleotide primers from the 16S-23S rRNA intergenic sequences for identifying different dairy and probiotic lactic acid bacteria by PCR. Intl J Food Microbiol 1997;35:49-56.

18. Dixon MF, Genta RM, Yardley JH, Correa P. Classification and grading of gastritis. The updated Sydney System. Am J Surg Pathol 1996; 20:1161-81.

19. Roberts P, Dickinson R, Whitehead A, Laughton C, Foweraker J. The culture of lactobacilli species in gastric carcinoma. J Clin Pathol 2002;55:477.

20. Ryan KA, O Hara AM, van Pijkeren J-P, Douillard FP, O Toole PW Lactobacillus salivarius modulates cytokine induction and virulence factor gene expression in Helicobacter pylori. J Med Microbiol 2009;58:996-1005.

21. Lesbros-Pantoflickova D, Corthésy-Theulaz I, Blum AL. Helicobacter pylori and probiotics. J Nutr 2007;137:812S-8S. 
22. González CG, Serrano C, Harris PR. Diagnóstico de la infección por Helicobacter pylori en niños mediante la detección de antígenos en deposiciones. Rev Med Chile 2007;135:182-8.

23. Ferreccio C, Rollán A, Harris PR, Serrano C, Gederlini A, Margozzini $\mathrm{P}$, et al. Gastric cancer is related to early Helicobacter pylori infection in a high-prevalence country. Cancer Epidemiol Biomarkers Prev 2007; 16:662-7.

24. Malfertheiner P, Megraud F, O'Morain C, Atherton J, Axon A, Bazzoli F, et al. Management of Helicobacter pylori infection-the Maastricht IV/ Florence Consensus Report. Gut 2012;61:646-64.

25. Otth L, Wilson M, Fernández H, Otth C, Toledo C, Cárcamo V, et al. Isolation of Helicobacter pylori in gastric mucosa and susceptibility to five antimicrobial drugs in southern Chile. Brazilian Journal of Microbiology 2011;42:442-7.

26. Konturek PC, Konturek SJ, BrzozowskI T. Helicobacter pylori infection in gastric cancerogenesis. J Physiol Pharmacol 2009;60:3-21.

27. Kusters JC, van Vliet AHM, Kuipers EJ. Pathogenesis of Helicobacter pylori Infection. Clin Microbiol Rev 2006;3:449-90.

28. Enroth H, Kraaz W, Engstrand L, Nyre n O, Rohan T. Helicobacter pylori strain types and risk of gastric cancer: A case-control study. Cancer Epidemiol Biomarkers Prev 2000;9:981-5.
29. Salgado F, Garcia A, Oñate A, González C, Kawaguchi F. Increased in-vitro and in-vivo biological activity of lipopolysaccharide extracted from clinical low virulence vacA genotype Helicobacter pylori strains. J Med Microbiol 2002;51:771-6.

30. García A, Barra R, Delgado C, Kawaguchi F, Trabal N, Montenegro $\mathrm{S}$, et al. Genotipificación de aislados clínicos de Helicobacter pylori en base a genes asociados a virulencia cagA, vacA y babA2. Primer aislamiento de una cepa babA2 positiva en pacientes chilenos. Rev Med Chile 2006;134:981-8.

31. Martínez A, González C, Kawaguchi F, Montoya R, Corvalán A, Madariaga J, et al. Helicobacter pylori: análisis de cagA y genotipificación de vacA en Chile. Detección de una cepa s $2 / \mathrm{m} 1$. Rev Med Chile 2001;129:1147-53.

32. Ortega JP, Espino A, Calvo A, Verdugo P, Pruyas M, Nilsen E, et al. Infección por Helicobacter pylori en pacientes sintomáticos con patología gastroduodenal benigna. Análisis de 5.664 pacientes. Rev Med Chile 2010;138:529-35.

33. Ryan KA, Jayaraman T, Daly P, Canchaya C, Curran S, Fang F, et al. Isolation of lactobacilli with probiotic properties from the human stomach. Lett Appl Microbiol 2008;47:269-74.

34. Mégraud F, Lehours P. Helicobacter pylori detection and antimicrobial susceptibility testing. Clin Microbiol Rev 2007;20:280-322. 